\section{FACTORES QUE INCIDEN EN LA DECISIÓN DE MOVILIDAD ESTUDIANTIL INTERNACIONAL}

\author{
FACTORS THAT INFLUENCE THE DECISION \\ OF INTERNATIONAL STUDENT MOBILITY
}

\section{Estela Maricela Villalón de la Isla | Antonio Humberto Closas Antonio Luzón Trujillo}

\section{RESUMEN}

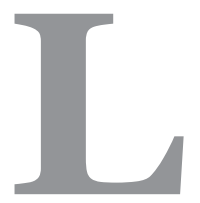

a movilidad es una de las principales estrategias del proceso de internacionalización de la Universidad de Guadalajara (UdeG), con lo cual, resulta de trascendental importancia conocer la percepción de sus estudiantes sobre los factores que inciden en la decisión de estudiar, o no, un semestre en otro país. Este estudio analiza la motivación y actitud de 126 estudiantes de Grado y Posgrado respecto a las posibilidades de movilidad internacional, desde su perspectiva. La muestra de participantes se conforma por alumnos activos matriculados en uno de los Centros Regionales que conforman la Red Universitaria de la UdeG: el Centro Universitario del Norte (CUNorte), ubicado en la ciudad de Colotlán, Jalisco, México. Entendida la movilidad estudiantil como una de las estrategias fundamentales en el proceso de Internacionalización de la Educación Superior, este trabajo contribuye al fortalecimiento de la investigación conjunta en temas que inciden en el cumplimiento de objetivos marcados en el eje temático de Internacionalización del actual Plan de Desarrollo del Centro Universitario.

Palabras clave: educación superior, internacionalización, movilidad estudiantil, movilidad internacional.

\section{ABSTRACT}

Mobility is one of the main strategies of the internationalization process of the University of Guadalajara (UdeG), which it is of transcendental importance to know the perception of its students about the factors that influence the decision to study, or not, a semester in another country. This study analyzes the motivation and attitude of 126 undergraduate and
Estela Maricela Villalón de la Isla mariceladelaisla@hotmail.com

Centro Universitario del Norte Universidad de Guadalajara

\section{MÉXICO}

Antonio Humberto Closas hclosas@hotmail.com

Facultad de Ciencias Económicas Universidad Nacional del Nordeste ARGENTINA

Antonio Luzón Trujillo aluzon@ugr.es

Facultad de Ciencias de la Educación Universidad de Granada

ESPAÑA 
graduate students regarding the possibilities of international mobility, from their perspective. The sample of participants is made up of active students enrolled in one of the Regional Centers that make up the University Network of UdeG, the University Center of the North (CUNorte), located in the city of Colotlán, Jalisco, Mexico. Understood student mobility as one of the fundamental strategies in the process of internationalization of Higher Education, this work contributes to the strengthening of research on issues that affect the achievement of objectives marked in the thematic axis of internationalization of the current Development Plan of the University Center.

Keywords: higher education, internationalization, student mobility, international mobility.

\section{INTRODUCCIÓN}

A escala global se observa un constante crecimiento de la movilidad estudiantil como parte de los procesos de Internacionalización de la Educación Superior. La inclusión de esta estrategia en las universidades se fortalece cada vez más con numerosos estudios e investigaciones sobre factores que definen la movilidad en determinadas regiones y contextos. Por su matrícula, la Universidad de Guadalajara es la segunda Institución Pública de Educación Superior más grande de México y la más importante de la región occidental del país. En el período 2016-2017 la cobertura de sus servicios educativos en el nivel medio superior representó el $45 \%$ del total estatal y el $34.8 \%$ en el nivel superior (Bravo, 2017). La Red Universitaria de la Universidad de Guadalajara (Red UdeG), se conforma por seis centros temáticos localizados en la zona metropolitana, un área geográfica donde se concentran alrededor de cinco millones de habitantes que representan el 61.9\% de la población total del estado (IIEG, 2017). Los centros regionales (nueve), se expanden estratégicamente en regiones potenciales de desarrollo socioeconómico. Partiendo de que la movilidad estudiantil es una de las principales formas de incluir la dimensión internacional en universidades en todo el mundo; aun cuando a escala mundial México no destaca por sus índices de movilidad, a nivel nacional, la Universidad de Guadalajara sí representa un referente para las universidades públicas del país. La UdeG se extiende a todo lo largo y ancho del estado de Jalisco en forma de Red (UdeG, 2017). A través del Centro Universitario Regional, CUNorte, atiende una de las regiones más desfavorecidas del estado. CUNorte es el contexto de la presente investigación, ya que la estratégica ubicación de este Centro Regional convierte a la UdeG en la principal institución de Educación Superior de la zona Norte de Jalisco. Su importancia trasciende más allá de la matrícula específicamente por la formación del capital humano indispensable para el desarrollo socioeconómico de la región. Dentro de la Red 
UdeG, CUNorte, ha sobresalido durante los últimos cuatro años por incrementar considerablemente sus índices de movilidad estudiantil internacional (Villalón de la Isla, 2017). Gran parte de la importancia de esta investigación radica en que la situación que se analiza no es un fenómeno aislado. El trabajo que aquí se presenta es el principio de una investigación que visualiza un extenso recorrido en el estudio de una problemática común en diferentes contextos. Este análisis es una propuesta concebida para extenderse, a través de un Centro Regional a toda la Red Universitaria de la Universidad Guadalajara, incluso más allá de sus fronteras en forma de proyecto de colaboración entre investigadores adscritos a universidades de diferentes países.

\section{MÉTODO}

\subsection{Objetivo}

El propósito principal de esta investigación es conocer las expectativas de los estudiantes del Centro Universitario del Norte (CUNorte) respecto a las posibilidades que tienen para estudiar un semestre en el extranjero. Se pretende identificar cuáles son los principales factores que inciden o limitan su decisión de solicitar ayudas y apoyos que el Centro Universitario oferta para la movilidad internacional como estrategia de internacionalización. Se trata de definir si la situación económica y familiar de los estudiantes es un factor que limita la movilidad internacional en el Centro Universitario.

Así, también se determinará qué aspectos académicos están relacionados con la oferta y acreditación de materias que dificultan la movilidad internacional estudiantil saliente.

\subsection{Población y Muestra}

La muestra a partir de la cual se realizaron los análisis estadísticos, que más adelante serán indicados, fue seleccionada durante el ciclo académico $2017 \mathrm{~B}$, utilizado básicamente el método aleatorio simple.

En concreto, la muestra elegida se encuentra formada por 126 alumnos, 78 mujeres (61.90\%) y 48 hombres (38.10\%), con una media $(M)$ de 22.55 años y desviación estándar (DE) de 6.13 .

Los estudiantes pertenecen a distintas carreras de Licenciaturas (111, 88.10\%) y Posgrados $(15,11.90 \%)$, que se imparten en el ámbito del Centro Universitario del Norte (CUNorte) de la Universidad de Guadalajara (UdeG), situado en la ciudad de Colotlán, Jalisco, México. Algunas de las características de la muestra utilizada en esta investigación, se ilustran en la Tabla 1. 


\begin{tabular}{c}
\hline ESTELA MARICELA VILLALÓN DE LA ISLA - ANTONIO HUMBERTO CLOSAS - ANTONIO LUZÓN TRUJILLO \\
FACTORES QUE INCIDEN EN LA DECISIÓN DE MOVILIDAD ESTUDIANTIL INTERNACIONAL \\
Págs. 25 - 47. DOI: $h t t p: / / d x$. doi.org/10.30972/rfce.0213726 \\
\hline
\end{tabular}

Tabla 1. Detalles relativos a la muestra empleada en la investigación empírica

\begin{tabular}{|c|c|c|c|}
\hline Institución & Nivel & Alumnos & Edad \\
\hline \multirow{3}{*}{ CUNorte-UdeG } & Licenciatura & $n=111(69 \mathrm{~m}, 42 \mathrm{~h})$ & $\begin{array}{l}\text { Mín. }=18 \text { Máx. }=37 \\
M=21.14\end{array}$ \\
\cline { 2 - 4 } & Posgrado & $n=15(09 \mathrm{~m}, 06 \mathrm{~h})$ & $\begin{array}{c}\text { Mín. }=19 \text { Máx. }=55 \\
M=33.00 \quad D E=9.39\end{array}$ \\
\hline \multicolumn{2}{|c|}{ Muestra: $N=126(78 \mathrm{~m}, 48 \mathrm{~h}) \mathrm{Edad}:$ Mín. $=18$, Máx. $=55, M=22.55, D E=6.13$} \\
\hline
\end{tabular}

Fuente: Elaboración propia

\subsection{Procedimiento de recogida de datos}

Una vez elegida la muestra, la recolección de los datos se llevó a cabo en una única instancia. En primer lugar, se les informó a los alumnos participantes que la aplicación del instrumento en cuestión respondía a un trabajo de investigación relacionado con el tema de movilidad académica internacional. También se les indicó sobre la importancia de responder sinceramente a los distintos ítems planteados, que sus respuestas tendrían un carácter estrictamente confidencial y que la participación en el estudio era una decisión totalmente voluntaria.

El momento temporal de este proceso fueron los meses de septiembre y octubre de 2017. La aplicación del cuestionario se realizó en un clima académico y contextual adecuado, y con el margen de tiempo necesario en virtud de las consultas formuladas en la prueba (20 minutos en promedio).

\subsection{Instrumento}

A efectos de evaluar distintos aspectos vinculados con la movilidad académica internacional de estudiantes del CUNorte-UdeG, se utilizó un cuestionario estructurado, en casi su totalidad. Dicho instrumento fue elaborado ad hoc por un equipo interdisciplinario de académicos, investigadores y profesionales, algunos de los cuales desarrollan sus actividades en el ámbito físico, social, cultural y educativo de los individuos que integran la muestra.

La prueba que se empleó (producción de datos primarios), esencialmente está compuesta por 41 ítems agrupados en cinco dimensiones:

1. Actitud (5 ítems), e.j., si tuviera oportunidad, estudiaría fuera de México.

2. Conocimiento (14 ítems), e.j., la página Web del CUNorte publica información sobre convocatorias para estudiar en el extranjero.

3. Aspectos familiares (10 ítems), ej., mi familia no está de acuerdo con la idea de que estudie en otro país.

4. Aspectos económicos (7 ítems), ej., no puedo estudiar en otro país por razones económicas.

5. Aspectos académicos ( 5 ítems), ej., no estudio un semestre en el extranjero porque significa atrasarme en mi carrera. 
Para la medición de las respuestas se ha utilizado una escala de tipo Likert, en la que las opciones fueron valoradas de 1 (totalmente en desacuerdo) a 5 (totalmente de acuerdo) puntos. Del total de ítems, 13 se encuentran formulados en sentido positivo (tal es el caso de los ejemplos proporcionados en las dos primeras dimensiones) y 28 en sentido negativo (como sucede con los ejemplos brindados en las tres últimas dimensiones).

Cabe indicar que las respuestas de aquellos ítems del cuestionario que tenían sentido negativo fueron revalorizadas de 1 (totalmente de acuerdo) a 5 (totalmente en desacuerdo), al momento de realizar los análisis estadísticos. De esta manera, en términos generales, puntajes directos (o promedios) altos en las respuestas denotarían mayor actitud, interés y predisposición de los estudiantes del CUNorte por la movilidad académica internacional. En cambio, puntajes directos (o promedios) bajos, ciertamente serían indicadores de todo lo contrario.

Además, con el propósito de conocer otras cuestiones también relevantes para la investigación, fueron elaborados de manera complementaria al cuestionario descrito en el párrafo anterior, cuatro ítems relacionados con: a) dominio de idioma inglés, b) tenencia de pasaporte, c) disponibilidad de visado americano, y d) interés de estudiar un semestre en el extranjero. Este último ítem, tiene particular importancia en el presente estudio debido a que será utilizado como variable respuesta (de tipo dicotómica, sí = o, no = 1) al momento de investigar el modelo logístico que mejor permita explicar o predecir la varianza de la misma a partir de las dimensiones del cuestionario sobre movilidad académica internacional (CuSMAI), que como se ha dicho fue elaborado especialmente para este estudio.

\subsection{Análisis de datos}

Comenzamos por señalar que la evaluación cualitativa del instrumento fue realizada por profesorado del Centro Universitario del Norte, CUNorte y del Centro Universitario de Ciencias Sociales y Humanidades, CUCSH, de la Universidad de Guadalajara, México, así como de la Corporación Universitaria COMFACAUCA, Colombia, en cuanto a los aspectos: a) pertinencia del contenido de los ítems propuestos (indicadores subjetivos de validez), y b) conformación del cuestionario en su conjunto (indicadores de la validez factorial o estructural), resultando favorable en ambos casos.

En efecto, las apreciaciones formuladas por el grupo de docentes que participaron en el análisis conceptual del test objeto de interés tuvieron un razonable porcentaje de coincidencia respecto del primer punto, mientras que, para la clasificación de los ítems en las cinco dimensiones de la encuesta, el porcentaje fue correcto. Los análisis realizados en la línea de validez cualitativa (juicio de expertos y grado de acuerdo, respectivamente), resultaron valiosos, a efectos de minimizar los márgenes de error del cuestionario al momento de su utilización en el espacio educativo propuesto para esta investigación.

De hecho, la validez de una prueba es un indicador del grado en que ésta es capaz de valorar lo que realmente pretende medir (Hernández, Fernández y Baptista, 2010), por lo que 
resulta necesaria y relevante su estimación cualitativa inicial, así como su evaluación cuantitativa en la etapa empírica de la investigación (análisis psicométricos).

Luego de realizar el proceso de detección y corrección en la base de datos, construida a partir de la información obtenida por la aplicación del cuestionario objeto de interés, se llevaron a cabo diferentes análisis pertenecientes al dominio de la psicometría (correlación dimensión-total corregida y consistencia interna), también de la estadística descriptiva (algunos estadísticos centrales y de dispersión) e inferencial (análisis correlacionales bivariados, análisis factorial, análisis de regresión logística y curva ROC; para las pruebas de hipótesis, utilizamos la medida $p$-valor).

Los diversos estudios indicados en el párrafo anterior fueron implementados con la intención de lograr los objetivos del presente trabajo, a través de: a) conocer el comportamiento de cada una de las dimensiones que componen la prueba aplicada, b) contrastar empíricamente la fiabilidad y validez del CuSMAI, y c) determinar la ecuación de predicción que mejor describa la relación entre el interés de estudiar un semestre en el extranjero (variable explicada o criterio) y las cinco dimensiones del CuSMAI (variables explicativas o predictoras). En todos los casos, el procesamiento de los datos fue realizado con ayuda del programa IBM SPSS Statistics 22.

\section{RESULTADOS}

En atención al propósito de esta investigación y a los análisis estadísticos anunciados en el apartado anterior, se presentan de forma sintética los resultados de aquellos indicadores que nos han parecido más convenientes calcular para: a) caracterizar la muestra en el total del cuestionario y en cada una de sus cinco dimensiones, b) conocer la asociación entre ellas, y con la variable criterio, c) distinguir la estructura factorial que resulta de la aplicación de la prueba, y d) modelizar la relación entre el interés de estudiar en el extranjero y las característica previstas en el CuSMAI.

\subsection{Estudios descriptivos y de fiabilidad de las dimensiones del cuestionario aplicado}

En la Tabla 2, pueden apreciarse los valores hallados para cada una de las dimensiones, así como para el conjunto de las mismas, en cuanto a puntuación directa, puntuación promedio, media y desviación estándar, los que resultaron razonables y se encuentran en el rango de medidas que se esperaban obtener. En puntuación promedio el valor medio más alto se obtuvo en la dimensión Actitud ( $M=4.11, D E=.79)$, en tanto que el menor valor medio resultó en la dimensión aspectos Económicos $(M=2.95, D E=1.15)$. La utilidad de estos estadísticos reside, 
entre otras cosas, en que nos ayudan a comprender de qué manera se encuentran distribuidos los datos de la muestra.

Tabla 2. Estadísticos descriptivos de las dimensiones del CuSMAI

\begin{tabular}{|c|c|c|c|c|c|c|c|}
\hline Dimensión & $\begin{array}{l}\text { No.de } \\
\text { ítems }\end{array}$ & $\begin{array}{c}\text { Puntuación } \\
\text { directa }\end{array}$ & Media & $D E$ & $\begin{array}{c}\text { Puntuación } \\
\text { promedio }\end{array}$ & Media & $D E$ \\
\hline Actitud & 5 & Mín. $=07$ Máx.$=25$ & 20.54 & 3.97 & Mín. $=1.4$ Máx. $=5$ & 4.11 & .79 \\
\hline Conocimiento & 14 & Mín. $=23$ Máx.$=68$ & 44.70 & 7.92 & Mín. $=1.6$ Máx. $=4.9$ & 3.19 & .92 \\
\hline Familiares & 10 & Mín. $=16$ Máx. $=50$ & 38.20 & 9.10 & Mín. $=1.6$ Máx. $=5$ & 3.82 & .56 \\
\hline Económicos & 7 & Mín. $=07$ Máx.$=35$ & 20.65 & 8.06 & Mín. $=1 \quad$ Máx. $=5$ & 2.95 & 1.15 \\
\hline Académicos & 5 & Mín. $=07$ Máx. $=25$ & 17.82 & 4.62 & Mín. $=1.4$ Máx. $=5$ & 3.56 & .92 \\
\hline \multicolumn{8}{|c|}{ CuSMAI (5 dimens. $=41$ ítems): P.D.Mín. $=90$ P.D.Máx. $=196 \quad M=142.16 \quad D E=22.26$} \\
\hline \multicolumn{8}{|c|}{ CuSMAI (5 dimens. $=41$ ítems): P.P.Mín. $=10.60$} \\
\hline
\end{tabular}

Fuente: Elaboración propia

Por otra parte, según se puede ver en la Tabla 3, las puntuaciones directas totales en cada una de las dimensiones muestran correlaciones corregidas aceptables con las puntuaciones directas totales en la prueba (sumatoria de los ítems que componen las dimensiones, excluidos aquellos que integran la dimensión cuya asociación se evalúa), puesto que en todos los casos superan el valor de referencia .20 (Kline, 2000), observándose la más alta (.52) en la categoría aspectos Familiares (luego siguen las dimensiones Actitud y Conocimiento, con valores .50 y .49, respectivamente). Este coeficiente, denominado índice de homogeneidad corregido, puede considerarse un indicador del grado de discriminación que posee la dimensión; cuanto más alta y positiva sea la correlación, mayor será la capacidad de la dimensión para discriminar los sujetos respecto del constructo o concepto que se pretende medir con la escala objeto de interés.

Respecto de los indicadores a de Cronbach cuando se excluye la dimensión (véase Tabla 3), podemos señalar que el menor valor hallado (.63) corresponde también a la categoría aspectos Familiares (luego siguen las dimensiones Conocimiento y aspectos Económicos, con valores .65 y .67, respectivamente). De cualquier manera, la totalidad de los coeficientes alfa encontrados verifican el criterio de algunos autores de hallarse por encima de .6o (Huh, Delorme y Reid, 2006; Nunnally, 1967), que es aplicable en un contexto de tipo exploratorio en las primeras fases de una investigación, como es nuestro caso.

Por lo tanto, puede sostenerse que la consistencia interna calculada para el cuestionario en cada una de las situaciones ( $\alpha$ de Cronbach sin la dimensión), ha resultado relevante y contribuye de manera favorable en la evaluación general de la fiabilidad del instrumento objeto de interés. Cabe mencionar que cuanto más bajo resulte este coeficiente alfa, siempre que no sea inferior al valor mínimo permitido, más se pone en evidencia el aporte de la dimensión a la consistencia interna del cuestionario (se recuerda que el coeficiente se calcula 
a partir de los ítems que conforman las restantes dimensiones; es decir, sin la participación de aquellos que pertenecen a la dimensión cuya contribución a la fiabilidad de la prueba se desea medir).

La fiabilidad, es una característica fundamental en cualquier test, y una de las formas de evaluarla es precisamente a través del coeficiente alfa de Cronbach, el cual indica la precisión o estabilidad de los resultados; señala la cuantía en que las medidas de la prueba están libres de errores casuales o aleatorios. En este sentido, la American Psychological Association (Wilkinson y Task Force on Statistical Inference, APA Board of Scientific Affairs (1999), American Psychological Association (2001)), también la política editorial de importantes revistas (Thompson, 1994), recomiendan calcular el coeficiente de fiabilidad en cada nueva muestra, y no apoyarse en la obtenida en otros estudios como aval de la fiabilidad del instrumento.

En atención a las consideraciones realizadas en párrafos anteriores, acerca del valor medio en puntuación promedio, así como de los coeficientes correlación dimensión-total corregida y a de Cronbach sin la dimensión, en puntuaciones directas, no cabe ninguna duda que las dimensiones Actitud y aspectos Familiares, son las que respectivamente poseen una mayor relevancia en el marco general del análisis descriptivo y de fiabilidad del CuSMAI.

Por último, según puede verse en la última fila de la Tabla 3, la consistencia interna calculada para el conjunto de las cinco dimensiones es correcta, puesto que el coeficiente alfa encontrado $(\alpha=.72)$ supera el criterio de .70 recomendado (Nunnaly y Bernstein, 1994).

Tabla 3. Estadísticos de fiabilidad de las dimensiones del CuSMAI

\begin{tabular}{|c|c|c|c|c|}
\hline Dimensión & $\begin{array}{c}\text { Media del } \\
\text { cuestionario } \\
\text { sin la dimensión }\end{array}$ & $\begin{array}{c}\text { DE del } \\
\text { cuestionario } \\
\text { sin la dimensión }\end{array}$ & $\begin{array}{c}\text { Correlación } \\
\text { dimensión-total } \\
\text { corregida }\end{array}$ & $\begin{array}{c}\text { a de Cronbach } \\
\text { del cuestionario } \\
\text { sin la dimensión }\end{array}$ \\
\hline Actitud & 121.46 & 20.28 & .50 & .68 \\
\hline Conocimiento & 97.44 & 17.79 & .49 & .65 \\
\hline Familiares & 103.79 & 16.59 & .52 & .63 \\
\hline Económicos & 121.62 & 17.89 & .45 & .67 \\
\hline Académicos & 124.33 & 20.31 & .41 & .70 \\
\hline & \multicolumn{2}{|c|}{ CuSMAI (5 dimens. $=41$ ítems): $\alpha$ de Cronbach $=.72$} \\
\hline
\end{tabular}

Fuente: Elaboración propia

Nota: El cálculo de los estadísticos de fiabilidad se realizó a partir de las puntuaciones directas de las dimensiones del CuSMAI.

\subsection{Análisis correlacionales bivariados}

En este apartado llevaremos a cabo análisis correlacionales entre las cinco dimensiones que integran el cuestionario, así como entre éstas y la variable interés de estudiar un semestre en el extranjero. La primera razón por la que se realizan estos estudios radica en el hecho de 
que los coeficientes que se obtengan permitirán en principio confirmar, o no, las relaciones que se presume existen entre las distintas variables que se analizan.

El segundo motivo reside en que está proyectado a continuación realizar un análisis factorial clásico con las dimensiones del CuSMAI (a efectos de evaluar la validez de constructo del instrumento) y es siempre de utilidad examinar previamente las relaciones lineales que las mismas presentan (en principio, con el fin de seleccionar adecuadamente el procedimiento de rotación que se empleará al momento de generar la matriz de pesos factoriales).

La tercera razón se debe a que las cuantificaciones que se logren permitirán elaborar una idea acerca del modelo logístico que sería posible diseñar con el objeto de explicar razonablemente las relaciones entre las dimensiones del cuestionario utilizado y el interés de estudiar un semestre en el extranjero.

En este marco, de acuerdo con los valores de la Tabla 4, puede afirmarse que la casi totalidad de las dimensiones (variables continuas) que componen el cuestionario correlacionan de manera positiva y estadísticamente significativa $(r=.20, p<.05 ; r=.23$ a .47, $p<.01)$. La correlación más alta se observa entre Actitud y aspectos Familiares, mientras que la más baja se presenta entre las variables, aspectos Familiares y aspectos Académicos. El único índice de correlación estadísticamente no significativo que fue hallado correspondió a las dimensiones Actitud y aspectos Económicos $(r=.10)$.

Por su parte, las correlaciones entre las diferentes categorías del CuSMAI y la variable interés de estudiar un semestre en el extranjero (variable dicotómica), varían de $\eta=.33$ (correlación baja) a $\eta=.69$ (correlación media). En este contexto, se distingue que los índices de mayor valor corresponden a las dimensiones Actitud $(\eta=.69)$ y aspectos Familiares $(\eta=.64)$.

Tabla 4. Matriz de correlaciones

\begin{tabular}{|c|c|c|c|c|c|c|}
\hline Variable & Actitud & Conocimiento & Familiares & Económicos & Académicos & Interés \\
\hline Actitud & 1 & $.35^{* * *}$ & $.47^{* *}$ & .10 & $.23^{* *}$ & .69 \\
\hline Conocimiento & & & $.23^{* *}$ & $.24^{* * *}$ & $.29^{* * *}$ & .55 \\
\hline Familiares & & & 1 & $\cdot 32^{* * *}$ & $.20^{*}$ & .64 \\
\hline Económicos & & & & 1 & $.24^{* * *}$ & .61 \\
\hline Académicos & & & & & 1 & .33 \\
\hline Interés & & & & & & 1 \\
\hline
\end{tabular}

Fuente: Elaboración propia $\quad{ }^{*} p<.05{ }^{* *} p<.01$

Nota: Para cuantificar el grado de relación lineal entre dos dimensiones del CuSMAI (variables continuas) se utilizó el coeficiente de correlación de Pearson (r). En cambio, para evaluar la asociación entre cada una de las dimensiones y el interés de estudiar un semestre en el extranjero (continua vs. dicotómica), empleamos el coeficiente de correlación Eta ( $\eta$ ).

Sería conveniente indicar que debido a que la casi totalidad de las cinco dimensiones del CuSMAI se encuentran con niveles significativos de correlación bivariada, hemos procedido a 
analizar la multicolinealidad (correlación entre tres o más variables independientes), a través de las medidas estadísticas tolerancia (T) y factor de inflación de la varianza (FIV). En realidad, lo que habitualmente está permitido es la presencia de multicolinealidad moderada; es decir, de una correlación mínima entre las covariables que conforman el modelo. Por el contrario, si la correlación fuese de mayor importancia, su efecto sería el incremento exagerado de los errores estándar y, en ocasiones, del valor estimado para los coeficientes de regresión, lo que ocasionaría por cierto que el poder predictivo de un posible modelo logístico se vea afectado. Sin embargo, es justo señalarlo, la multicolinealidad no hará que aparezca significación estadística en aquellas estimaciones donde no existe, y viceversa.

$\mathrm{Al}$ respecto debemos señalar que cuando se utilizaron los dos procedimientos analíticos indicados (Ty FIV) los valores obtenidos fueron correctos, en el sentido que no revelaban ningún grado de multicolinealidad (en virtud de que SPSS no aporta índices de multicolinealidad para la regresión logística, hemos utilizado sendos modelos de predicción lineal múltiple en los que una de las covariables actuaba como variable dependiente y las restantes covariables como variables independientes de aquella). En efecto, los estadísticos T resultaron entre .66 a .91, superando en todos los casos el valor de referencia .40; mientras que las medidas FIV se encontraban entre 1.10 y 1.51, esto es, claramente alejadas de 10. Las referencias que hemos citado surgen del consenso general teórico y empírico que tradicionalmente se emplea para detectar la existencia de multicolinealidad entre los regresores de un modelo logístico.

Antes de pasar al siguiente análisis estadístico, sería conveniente detenernos en la observación de un detalle, puesto que podría ayudarnos a comprender mejor la ecuación de predicción, que será analizada más adelante, entre las cinco dimensiones del CuSMAI (variables predictoras) y el interés de estudiar un semestre en el extranjero (variable explicada). En efecto, nos referimos al hecho de que en el estudio descriptivo y de confiabilidad de las dimensiones, así como en el análisis de correlaciones entre las categorías del cuestionario y la variable interés, las dos dimensiones que repetidamente se revelaron destacadas y libres de objeciones para conformar la ecuación de regresión en el rol de variables explicativas, fueron Actitud en primer lugar y aspectos familiares en segundo término.

\subsection{Análisis factorial}

Si bien, en una aproximación teórica y empírica (véase Tabla 4) puede decirse que las dimensiones medidas se encontrarían agrupadas en determinados factores (e.j., Actitud y aspectos Familiares conformarían un factor; Conocimiento, junto con aspectos Económicos y aspectos Académicos, integrarían otro factor), emplearemos la técnica del análisis factorial a efectos de identificar claramente estas variables latentes; es decir, contrastar la validez de constructo o estructural del cuestionario aplicado.

Siguiendo a Comrey (1973), antes de realizar el análisis factorial clásico, se ha comprobado la adecuación de los datos para este tipo de estudio. Una síntesis de los resultados es la 
siguiente: a) determinante de la matriz de correlaciones (.46) relativamente bajo; b) medida de adecuación de la muestra de Kaiser, Meyer y Olkin, índice $K M O=.70$, ubicado en un rango que se considera aceptable de potencial explicativo de las variables (Kaiser, 1974); c) contraste de esfericidad de Bartlett con $\chi^{2}(10)=73.59, p=$.oo. En vista de los valores obtenidos, en principio, el análisis factorial es una opción pertinente que nos ayudará a identificar los factores subyacentes al grupo de cinco dimensiones que se analizan.

Para determinar la solución factorial hemos empleado el criterio del porcentaje de varianza explicada; esto es, se procedió a extraer aquel número de factores que permita explicar al menos el 60\% de la varianza total de los datos. Si bien no está determinado un porcentaje preciso al respecto, algunos autores señalan que para investigaciones que se realizan en áreas de Ciencias Sociales lo recomendable es que la varianza acumulada no sea inferior al 50\% (Henson y Roberts, 2006).

Finalmente, la extracción se realizó por el método de componentes principales y rotación varimax, en atención a los resultados obtenidos en los análisis correlacionales. El conjunto de decisiones adoptadas ha delimitado efectivamente una estructura bifactorial que explica un 61.14\% de la varianza total, lo que puede interpretarse como una solución coherente y un porcentaje correcto, en virtud de las distintas apreciaciones efectuadas en los párrafos anteriores.

Según los resultados del análisis realizado el factor que permite explicar la mayor cantidad del total de la varianza de la muestra está integrado por las dimensiones Conocimiento, aspectos Económicos y aspectos Académicos (42.09\%), mientras que el segundo factor se encuentra conformado por las áreas Actitud y aspectos Familiares (19.05\%).

En la Tabla 5 se recogen, para cada uno de los factores subyacentes, el valor propio, el porcentaje de varianza tanto explicada como acumulada, las dimensiones que los integran, sus comunalidades (proporción de varianza de la variable que puede ser explicada por el modelo factorial obtenido) y finalmente sus cargas factoriales o saturaciones (correlación entre la variable y el factor).

Tabla 5. Análisis factorial por componentes principales y rotación varimax

\begin{tabular}{|c|c|c|c|c|c|c|}
\hline Factor & Dimensión & $\begin{array}{c}\text { Valor } \\
\text { propio }\end{array}$ & $\begin{array}{c}\text { Varianza } \\
\text { explicada }\end{array}$ & $\begin{array}{c}\text { Varianza } \\
\text { acumulada }\end{array}$ & Comunalidad & Saturación \\
\hline \multirow{2}{*}{$\begin{array}{c}\text { Macrosociológico } \\
\text { Económicos }\end{array}$} & $\begin{array}{c}\text { Conocimiento } \\
\text { Académicos }\end{array}$ & 2.11 & 42.09 & 42.09 & .69 & .51 \\
.40 & .63 & .65 & .59 \\
\hline Microsociológico & $\begin{array}{c}\text { Actitud } \\
\text { Familiares }\end{array}$ & 1.02 & 19.05 & 61.14 & .61 & .72 \\
\hline
\end{tabular}

Fuente: Elaboración propia

Evidentemente, en atención a lo que antecede, podemos señalar que el modelo factorial ha verificado un aspecto que es estratégico en toda acción de modelado estadístico: la "parsi- 
monia"; esto es, explicación más simple entre varias posibles. En efecto, la estructura obtenida, por un lado, posee un reducido número de factores y, por otro, ambos son susceptibles de interpretación sustantiva.

Así pues, el Factor 1, se halla integrado por aquellas dimensiones que se encuentran correlacionadas entre si y se caracterizan por incluir en sus ítems diversas expresiones sobre movilidad académica asociadas con el Conocimiento de opciones, también con aspectos tanto Económicos como Académicos de la temática objeto de estudio, lo que le confiere a esta variable latente (i.e., Factor 1), y en este marco de trabajo, un perfil más bien cercano a cuestiones que podrían llamarse de tipo macrosociológicas.

Por su parte, el Factor 2, se encuentra conformado por dos dimensiones que además de estar fuertemente asociadas $(r=.47, p<.01)$, sus ítems hacen referencia a distintas situaciones vinculadas con la Actitud hacia la movilidad académica y con aspectos Familiares asociados con la misma, por lo que los rasgos de la actual construcción teórica (i.e., Factor 2), en este contexto de operaciones, estarían en la línea de temas relativos a teorías microsociológicas.

En síntesis, los distintos indicadores obtenidos a lo largo de esta investigación nos llevan a concluir que el cuestionario bajo estudio es fiable y presenta validez de constructo; esto es, sería de utilidad para medir razonablemente el interés de los estudiantes de CUNorte-UdeG respecto de la movilidad académica internacional.

\subsection{Análisis de regresión logística}

En vista del tercer objetivo planteado en este estudio y de los coeficientes obtenidos en el análisis correlacional, se ha considerado adecuado emplear en la estimación del modelo de regresión logística el método "Introducir", disponible en IBM SPSS Statistics 22. El procedimiento citado es recomendable en aquellos casos que el investigador se halla interesado en conducir el estudio en función de los resultados que va logrando, tal como sucede en esta ocasión.

Se recuerda, además, que los propósitos del modelo logístico residen en determinar la existencia o ausencia de relación entre una o más variables independientes y la variable dependiente; medir la magnitud de dicha relación y explicar o predecir la probabilidad de que la variable criterio sea igual a 1, en función de los valores que adopten las variables predictoras (Jovell, 1995).

En atención a lo expresado, ha sido ingresada como variable respuesta el interés de estudiar un semestre en el extranjero ( $s i ́=0$, no = 1), y como variables explicativas o covariables las cinco dimensiones: Actitud, Conocimiento, Familiares, Económicos y Académicos del CuSMAI.

En virtud de las opciones elegidas y de los datos de la muestra, se han obtenido para las variables independientes introducidas los valores que se muestran en la Tabla 6 (coeficientes $B$ del modelo, desviaciones estándar, estadísticos de Wald, $p$-valores asociados). 
Tabla 6. Coeficientes estimados y estadísticos relacionados (cinco dimensiones)

\begin{tabular}{|c|c|c|c|c|}
\hline Variable & $B$ & $D E$ & Wald & Valor $\boldsymbol{p}$ \\
\hline Actitud & -0.35 & 0.10 & 12.25 & .00 \\
\hline Conocimiento & 0.11 & 0.08 & 1.89 & .25 \\
\hline Familiares & -0.14 & 0.07 & 4.02 & .04 \\
\hline Económicos & -0.16 & 0.07 & 4.97 & .03 \\
\hline Académicos & 0.10 & 0.09 & 1.23 & .38 \\
\hline
\end{tabular}

Fuente: Elaboración propia

Puede observarse en la Tabla 6 que los $p$-valores asociados a los estadísticos de Wald (contrastan la hipótesis nula de coeficiente igual a cero) inferiores a .05 son los relativos a los coeficientes: $B 1=-0.35(p=.00)$, de la variable predictora Actitud; $B 3=-0.14(p=.04)$, de la variable predictora aspectos Familiares; y $B 4=-0.16(p=.03)$, de la variable predictora aspectos Económicos. En virtud de lo expresado, así como de otros criterios que fueron asumidos a partir de indicadores que no se encuentran en la tabla (e.g., $\operatorname{Exp}(B)$ y sus respectivos intervalos de confianza al 95\%), hemos considerado razonable sostener que las variables Actitud, aspectos Familiares y aspectos Económicos, serían relevantes a la hora de explicar o predecir la variabilidad del interés de estudiar un semestre en el extranjero (entiéndase en un futuro cercano al momento de aplicación del cuestionario).

Así pues, es que se procedió a plantear un modelo logístico binario conformado sólo por las tres dimensiones mencionadas en el párrafo precedente como únicas covariables de la ecuación; los distintos valores obtenidos pueden apreciarse en la Tabla 7. Según puede verse, la totalidad de los $p$-valores (varían de .oo a .04), asociados a los respectivos estadísticos de Wald, resultaron inferiores a .05, tal como se suponía que ocurriría.

Tabla 7. Coeficientes estimados y estadísticos relacionados (cinco dimensiones)

\begin{tabular}{|c|c|c|c|c|}
\hline Variable & $B$ & $D E$ & Wald & Valor $\boldsymbol{p}$ \\
\hline Actitud & -0.37 & 0.09 & 16.90 & .00 \\
\hline Familiares & -0.15 & 0.06 & 6.25 & .03 \\
\hline Económicos & -0.11 & 0.05 & 4.84 & .04 \\
\hline Constante & 15.50 & 3.95 & 15.40 & .00 \\
\hline
\end{tabular}

Fuente: Elaboración propia

De manera que, para el nivel de significación adoptado, $\alpha=.05$, queda firme el rechazo de la hipótesis nula y, en consecuencia, concluimos que las tres covariables analizadas serían útiles para explicar/predecir la varianza de la variable interés (cabe recordar que la evaluación de la significatividad estadística debe realizarse sobre los coeficientes $B$ asociados a las covariables, y no sobre la constante; la que es incorporada en la ecuación con el objeto de que el modelo en su conjunto logre un mejor ajuste a los datos que fueron usados para estimarlo). 
Llegados a este punto, se considera oportuno señalar la importancia de realizar una correcta interpretación del signo de los coeficientes de regresión $(B)$ y de su relación con los valores de la variable que se pretende explicar.

En efecto, el signo negativo en cada uno de los coeficientes $B$ de las covariables Actitud, Familiares y Económicos -se recuerda que sus posibles respuestas oscilan entre 1 (totalmente en desacuerdo) y 5 (totalmente de acuerdo) puntos-, significa que, en la medida que aumentan los valores de estas variables y, por lo tanto, se eleva el nivel de Actitud, así como el respaldo de Familiares y la disponibilidad de recursos Económicos, descienden los valores de la variable respuesta, que como se dijo es de tipo dicotómica y varía entre o (sí tiene interés) y 1 (no tiene interés). Dicho de otra manera, puntajes altos en las respuestas a las variables independientes, producirá en la variable dependiente una escasa probabilidad de ocurrencia del evento; por lo tanto, el modelo que será propuesto a continuación clasificará a estos sujetos en el grupo de estudiantes del CUNorte con interés por la movilidad académica internacional.

Por cierto, si las respuestas en las covariables se corresponden con valores bajos, la variable dependiente tendrá una probabilidad elevada (i.e., más cercana a 1 que a o), de forma que el modelo logístico clasificará a estos jóvenes en el grupo de alumnos con escaso interés por la movilidad académica internacional.

Ahora sí, a partir de la estimación de los coeficientes de las variables explicativas Actitud, Familiares y Económicos, y del correspondiente análisis de significatividad estadística de cada uno de ellos (véase Tabla 7), es posible desarrollar la ecuación de regresión correspondiente al modelo logístico, la que se presenta a continuación:

$p($ Interés $=$ No $)=\frac{1}{1+\operatorname{Exp}(-15.50+0.37 \times \text { Actitud }+0.15 \times \text { Familiares }+0.11 \times \text { Económicos })}$ Fuente: Elaboración propia

Con respecto al contraste global para el modelo que se acaba de proponer, podemos indicar que, en el test de bondad de ajuste, según puede observarse en la Tabla 8, el $p$-valor asociado al estadístico $\chi^{2}(3)=41.57$ resultó igual a .oo. Por lo tanto, para el nivel de significación $\alpha=.05$, se rechaza la hipótesis nula de que todos los coeficientes de las variables incluidas en el modelo son iguales a cero.

A su vez, la prueba de Hosmer-Lemeshow (contrasta la hipótesis nula de que el modelo propuesto puede explicar lo que se observa), otra forma de evaluar la bondad de ajuste de un modelo de regresión logística, ha proporcionado un $p$-valor de .12 (mayor que $\alpha=$ .05, por lo que no debe rechazarse la hipótesis nula), para el estadístico Chi-cuadrado con 8 grados de libertad, cuya medida resultó 12.67; de manera que, en sintonía con lo expresado en el párrafo anterior, podemos sostener que el modelo que se propone se ajusta a los datos empíricos. 
Tabla 8. Indicadores globales del modelo

\begin{tabular}{|c|c|c|c|}
\hline Test & $\boldsymbol{\chi}^{\mathbf{2}}$ & $\boldsymbol{g l}$ & Valor $\boldsymbol{p}$ \\
\hline Bondad de ajuste & 41.57 & 3 & .00 \\
\hline Hosmer-Lemeshow & 12.67 & 8 & .12 \\
\hline
\end{tabular}

Fuente: Elaboración propia

Con el objeto de traducir en forma práctica la utilización del modelo propuesto, se presenta de inmediato la siguiente situación particular. En efecto, si se aplicara la ecuación de regresión a los datos de estudiantes que poseen puntuaciones directas iguales o superiores al valor medio en las dimensiones Actitud (20.54), Familiares (38.20) y Económicos (20.65), se podría predecir que tendrían interés de estudiar un semestre en el extranjero. Esto es así, puesto que se lograrían probabilidades, según el modelo planteado, iguales o inferiores al 47.50\%; es decir, más cercanas a o que a 1, de modo que los alumnos en cuestión deberían ser clasificados en el grupo de jóvenes que sí estarían interesados en estudiar fuera de México.

Por el contrario, en aquellos estudiantes con puntajes directos bajos (inferiores a sus respectivos valores medios) en las variables Actitud, Familiares y Económicos (o en alguna de ellas), se observaría un menor o escaso interés de estudiar un semestre en el extranjero. Desde luego, en estos casos la tendencia sería que a través del modelo propuesto se obtengan probabilidades superiores al 50\%, por lo que estarían más próximas a 1 (no tiene interés) que a o (sí tiene interés).

\subsection{Análisis de la curva ROC}

Con el objeto de mostrar la capacidad global que el modelo logístico posee para explicar o predecir el interés de estudiar un semestre en el extranjero, así como de elegir el punto de corte más apropiado para una sensibilidad o una especificidad determinada, utilizaremos el concepto de la curva ROC (Receiver Operating Characteristic).

La sensibilidad indica la capacidad del estimador (modelo propuesto) para identificar o clasificar correctamente los casos positivos, de entre todos los casos positivos disponibles durante la prueba (en este estudio, los casos positivos se relacionan con jóvenes que carecen de interés en estudiar en el extranjero). Mientras que, la especificidad es la capacidad del estimador para detectar correctamente los casos negativos, de entre todos los casos negativos disponibles durante la prueba (en esta investigación, los casos negativos se vinculan con alumnos que poseen interés de estudiar fuera de México).

Quizás sería conveniente comentar que, en general, se adoptan como casos positivos aquellos eventos en los que se encuentra presente la problemática objeto de análisis (alumnos sin interés por realizar estudios en el extranjero). Por el contrario, se adoptan como casos negativos aquellos sucesos en los que está ausente la cuestión objeto de estudio (alumnos con interés por estudiar en el exterior). 
En este contexto, se presentan e interpretan a continuación los resultados más relevantes, a partir de los datos muestrales y utilizando como variable de contraste las probabilidades generadas por el modelo propuesto, mientras que la variable de estado será el interés de estudiar un semestre en el extranjero.

En la Tabla 9 pueden apreciarse diferentes valores del área bajo la curva ROC. La estimación puntual ha resultado igual a .85 (mínimo exigible .50). El desvío estándar de esta estimación vale .05, valor que multiplicado por 1.96 (corresponde para un nivel de confianza del 95\%), luego restado y sumado de .85 , nos proporciona respectivamente el límite inferior (.75) y el límite superior (.95) del intervalo de confianza. Como este intervalo no contiene al valor .50, podemos afirmar que el área bajo la curva ROC de nuestro estudio es significativamente mayor que el mínimo exigible para un método de diagnóstico; lo que se halla confirmado por la significación asintótica (.oo), que no es más que el $p$-valor del test.

En definitiva, podemos rechazar la hipótesis nula [AUC (Área Under the Curve) $=.50$ ], y concluir que la estimación puntual del área bajo la curva ROC (.85) estaría indicando que el modelo logístico (conformado por los predictores Actitud, Familiares y Económicos), que se está utilizando como variable de contraste, posee una calidad diagnóstica correcta para clasificar el interés de los estudiantes de la muestra.

Tabla 9. Área bajo la curva ROC

\begin{tabular}{|c|c|c|c|c|}
\hline \multirow{2}{*}{ Área } & \multirow{2}{*}{ DE } & \multirow{2}{*}{ Valor $p$} & \multicolumn{2}{|c|}{ IC al 95\% para el área } \\
\cline { 4 - 5 } & & & Inferior & Superior \\
\hline .85 & .05 & .00 & .75 & .95 \\
\hline
\end{tabular}

Fuente: Elaboración propia

En la Figura 1, puede apreciarse la representación gráfica de la curva ROC ajustada a los datos muestrales. En este caso, la curva está razonablemente por encima de la recta $y=x$, por lo que podemos considerar que el método de diagnóstico es aceptable para discriminar el interés de los estudiantes. La flecha indica el punto de corte (.70) que determina la sensibilidad (.80) y especificidad $(1-.20=.80)$ conjuntas más alta (Índice de Youden $=.80+.80-1=.60$ ).

Así, por ejemplo, podríamos indicar que para una sensibilidad del $80 \%$, que se consigue en el punto de corte .70 (probabilidad que podría lograrse utilizando en las covariables puntuaciones directas, por ejemplo, de 19.6 en Aptitud, 33.4 en Familiares y 21.7 en Económicos), tendríamos una especificidad de $1-.20=.80$ (en el área de Ciencias Sociales, valores de especificidad superiores a .70 se consideran aceptables).

Esta situación sería la más conveniente, puesto que frecuentemente lo que desea obtener es aquel punto de corte que genere, al mismo tiempo, los mayores porcentajes de sensibilidad y especificidad (en este caso se dice que presenta el mayor índice de Youden, calculado según la fórmula: Sensibilidad + Especificidad - 1). 




Figura 1. Gráfico del análisis de la curva ROC

No obstante, dicho punto de corte no necesariamente determina la sensibilidad ni la especificidad más alta que podría alcanzar el test (generalmente están determinadas por puntos de corte distintos). De hecho, existen situaciones en las que se requiere disponer de un test diagnóstico altamente sensible o bien altamente específico. En tales circunstancias, no es aconsejable utilizar el punto de corte identificado por el índice de Youden; por el contrario, resulta más útil conocer los valores de sensibilidad y especificidad determinados por diferentes puntos de corte, y optar por aquel que responda al objetivo buscado.

Todas estas apreciaciones se realizan en el marco de las posibilidades (fortalezas y debilidades) que el modelo posee para explicar y/o predecir la varianza del interés de los individuos de la muestra, a partir de las puntuaciones en las dimensiones que participan. Por lo tanto, estas estimaciones e interpretaciones deben ser consideradas con debida prudencia, en razón del particular escenario empírico en el que se ha desarrollado el trabajo y de la metodología de abordaje que ha sido implementada, circunstancias que indudablemente limitan las bondades que la presente investigación pudiera ofrecer.

Sin embargo, en atención a que el número de aciertos globales del modelo es uno de los indicadores más importante de la bondad de ajuste del mismo, podemos sostener que la ecuación estimada resulta razonable para clasificar y predecir las categorías de la variable dependiente. En efecto, lo anterior se apoya en el hecho de, si se aplicara el modelo propuesto a las observaciones muestrales, se obtendrían porcentajes de éxitos del 80\%, según ha sido anticipado.

Finalizamos la presente sección indicando: a) los estudios psicométricos desarrollados en los aparatados primero y tercero, permitieron sostener que el CuSMAI, es un instrumento confiable y que posee validez de constructo; b) los análisis descriptivos y correlacionales, llevados a cabo en los dos primeros apartados, proporcionaron información coherente con el 
modelo logit obtenido; c) mediante la utilización de la ecuación de regresión, pudo comprobarse que los estudiantes que obtuvieron puntajes directos iguales o superiores a la media en las variables Aptitud, Familiares y Económicos, tendrían interés de estudiar fuera de México, ya que obtendrían probabilidades iguales o inferiores al punto de corte (.70), determinado a través del análisis de la curva ROC; y d) fue posible contrastar empíricamente la validez explicativa, predictiva y discriminante del CuSMAI respecto de la variable interés, en especial de las dimensiones que participan como covariables del modelo.

\section{DISCUSIÓN Y CONCLUSIONES}

En el presente estudio nos habíamos propuesto concretar, en un dominio estadístico de tipo psicométrico, descriptivo e inferencial, diferentes acciones, las cuales básicamente estaban relacionados con demostrar la fiabilidad y validez del CuSMAI, así como con determinar la ecuación de predicción que mejor reflejara la relación entre el interés de los jóvenes del CUNorte de realizar un semestre académico en el extranjero y las cinco dimensiones del cuestionario aplicado. Pues bien, en vista de los resultados obtenidos en el marco de esta investigación, y de las consideraciones que se presentan a continuación, podríamos sostener que las acciones planteadas han sido logradas.

En efecto, el análisis de los datos que derivaron de la aplicación del CuSMAI, generó la posibilidad de contar con información directa del espacio académico de selección de la muestra. Este hecho es, sin duda, relevante puesto que permite, a partir de los resultados, brindar mejores explicaciones acerca de los factores que inciden en el interés y la decisión de los estudiantes por estudiar fuera de su país de origen, posibilitando a posteriori adoptar medidas de intervención más ajustadas y eficientes con el propósito final de mejorar los bajos índices de movilidad internacional en este centro universitario.

A partir de algunos estudios iniciales (media, desviación estándar, correlación dimensión-total y alfa de Cronbach), realizados sobre el cuestionario utilizado y sus cinco dimensiones, así como de los análisis implementados posteriormente (correlaciones bivariadas, multicolinealidad, análisis factorial, regresión logística y curva ROC), fue posible esencialmente: a) comprobar que el cuestionario aplicado constituye un instrumento fiable y válido; b) evaluar las correlaciones entre las dimensiones del cuestionario, así como entre éstas y la variable interés; y c) obtener una ecuación de regresión que permita explicar y clasificar aceptablemente el interés de los estudiantes de la muestra de estudiar en el exterior.

Si bien los resultados logrados indican ciertas evidencias acerca de las bondades de este estudio, creemos necesario señalar algunas limitaciones que hemos observado. 
En primer lugar, los sujetos de la presente investigación fueron alumnos pertenecientes a un centro académico específico, lo que quizás no permite hacer inferencias demasiado amplias sobre otros estudiantes universitarios, menos aún extender los resultados obtenidos sobre otras poblaciones no representadas en la muestra.

En segundo término, el modelo que se propone sólo cuenta con tres de las cinco posibles variables predictoras, lo que probablemente se deba a que la influencia de éstas sobre el interés de los estudiantes por la movilidad internacional, no es exclusivamente directa. En efecto, podría pensarse que en realidad la capacidad explicativa y predictiva de un modelo verdaderamente ajustado y representativo, se encuentra mediatizada por otras variables, especialmente de tipo motivacionales, afectivas y contextuales, que influyen en el interés, formando así, posiblemente, un entramado de relaciones directas, indirectas y recíprocas.

A pesar de lo expuesto, por lo que los resultados alcanzados deberían considerarse con cierta cautela, pensamos que el estudio realizado debe ser reconocido como un paso adelante en el abordaje del tema objeto de interés y, consecuentemente, un aporte a la comunidad científica y profesional del área de conocimiento, con posibles proyecciones en política, planificación y gestión educativa.

El trabajo llevado a cabo nos hizo ver con interés el desarrollo de futuras investigaciones en torno a los siguientes temas: a) utilizando otros determinantes de la variable interés, además de las consideras en este estudio, elaborar un modelo causal y probar su validez de medida y global, empleando la técnica multivariante denominada estructuras de covarianza; b) replicar el actual estudio en intervalos de tiempo determinados (semestrales o anuales), con el fin de obtener información sobre los posibles efectos o cambios que se presentan en el interés de los estudiantes por la movilidad internacional, debido al incremento en la edad, a la adquisición de nuevas competencias, y al relato de otros estudiantes que hayan transitado la experiencia de estudiar fuera de México; y c) llevar a cabo trabajos de similares características al presente en los que sea posible realizar comparaciones cuantitativas entre distintas variables, tales como el género de los estudiantes, el tipo de carrera que siguen, el nivel de estudios (licenciatura o posgrado) en el que se encuentran, entre otros factores.

Como última reflexión, se indica que el hecho de haber logrado un legítimo modelo empírico en un determinado contexto académico y sociocultural (estudiantes de distintas carreras y niveles de un centro universitario de gestión pública, ubicado en la región norte de Jalisco, México), da origen a contar con un primer marco de referencia en el tema objeto de estudio.

Asimismo, permite sostener que la adopción de la metodología utilizada ha sido una decisión correcta y que el cuestionario aplicado puede considerarse un instrumento de medición sustentable. No obstante, respecto de este último aspecto, debemos señalar que el tratamiento estadístico de los datos nos permitió observar la conveniencia de que al menos la mitad de los ítems que se encuentran formulados en sentido negativo, sean expresados en sentido positivo, sin alterar, lógicamente, el significado y la dirección que originalmente poseen en el CuSMAI. 
Desde nuestro punto de vista, propiciar pautas de intervención sobre los factores que se encuentran asociados al interés de los jóvenes de estudiar en el extranjero, representa una tarea relevante debido a que los resultados de participación en actividades de movilidad internacional tienen una implicación en la evolución personal y en el desempeño académico. Estos efectos, por sí mismos, son suficientes para pensar favorablemente en la conveniencia de incrementarse sus líneas de investigación, con el fin de lograr un mayor desarrollo sobre su conocimiento.

Efectivamente, extender y profundizar acciones de indagación sobre la temática de cómo mejorar los bajos índices de movilidad internacional, tanto en CUNorte, como en otros centros universitarios que dependen de la UdeG, sería una importante contribución a la comunidad académica de su zona de influencia. Esto es así, en virtud de que en última instancia se favorecería el rendimiento de los alumnos, que como es sabido constituye una de las principales preocupaciones de los sistemas educativos estatales en la mayoría de los países y regiones de América Latina.

\section{REFERENCIAS BIBLIOGRÁFICAS}

American Psychological Association (2001). Publication Manual of the American Psychological Association. Washington DC: Author.

Bravo, I. T. (2017) Estadística institucional 2016-17. Guadalajara, México: Universidad de Guadalajara. Obtenido de: http://www.rectoria.udg.mx/sites/default/files/IA2016estadisticainstitucionalTBP.pdf

Comrey, A. L. (1973). A First Course in Factor Analysis. New York: Academic Press.

Henson, R. K. y Roberts, J. K. (2006). Use of Exploratory Factor Analysis in Published Research: Common Error and Some Comment on Improved Practice. Educational and Psychological Measurement, 66(3), 393-416.

Hernández-Sampieri, R., Fernández, C., y Baptista, P. (2010). Metodología de la investigación (5a. ed.). México, DF: McGraw-Hill.

Huh, J., Delorme, D. y Reid, L. (2006). Perceived third-person effects and consumer attitudes on preventing and banning DTC advertising. Journal of Consumer Affairs, 4O(1), 90-116. 
Instituto de Información Estadística y Geográfica. IIEG (2017). Población en Jalisco 2017. Strategos. Revista Digital de Información Estadística y Geográfica. Obtenido de: https://iieg. gob.mx/strategos/portfolio/poblacion-en-jalisco-2017/

Jovell, A. J. (1995). Análisis de Regresión Logística. Madrid: Centro de Investigaciones Sociológicas.

Kaiser, Henry F. (1974). An index of factorial simplicity. Psychometrika, 39, 31-36.

Kline, Paul (2000). The Handbook of Psychological Testing (2nd.ed.). London: Routledge.

Nunnally, J. C. (1967). Psychometric Theory. New York: McGraw-Hill.

Nunnaly, J. y Bernstein, I. H. (1994) Psychometric Theory (3rd. ed.). New York: McGraw-Hill.

Thompson, B. (1994). Guidelines for authors. Educational and Psychological Measurement, 54, 837-847. Universidad de Guadalajara, UdeG (2017). Red Universitaria de Jalisco. Obtenido de: http://www.udg.mx/es/red-universitaria

Villalón de la Isla, E. M. (2017). La movilidad estudiantil en el proceso de internacionalización. Estrategias metodológicas para su estudio. Revista Española de Educación Compara$d a, 29,297-314$.

Wilkinson, L. y Task Force on Statistical Inference, APA Board of Scientific Affairs (1999). Statistical Methods in Psychology Journals: Guidelines and Explanations. American Psychologist, 54(8), 594-604.

\section{COMO CITAR ESTE ARTÍCULO}

VILLALÓN DE LA ISLA, Estela Maricela, CLOSAS, Antonio Humberto, LUZÓN TRUJILLO, Antonio. Factores que inciden en la decisión de movilidad estudiantil internacional. Revista de la Facultad de Ciencias Económicas - UNNE, Argentina. Volumen 21 Núm. 2, julio-diciembre 2018, ISSN 1668 - 6365. Págs. 25 - 47. DOI: http://dx.doi.org/10.30972/rfce.0213726 


\begin{tabular}{c}
\hline ESTELA MARICELA VILLALÓN DE LA ISLA - ANTONIO HUMBERTO CLOSAS - ANTONIO LUZÓN TRUJILLO \\
FACTORES QUE INCIDEN EN LA DECISIÓN DE MOVILIDAD ESTUDIANTIL INTERNACIONAL \\
Págs. 25 - 47. DOI: $h$ ttp://dx.doi.org/10.30972/rfce.0213726 \\
\hline
\end{tabular}

\section{CURRICULUM VITAE}

\section{Estela Maricela Villalón de la Isla}

Se desempeña como Profesora Asociada de Tiempo Completo y Responsable del Área de Internacionalización del Centro Universitario del Norte, de la Universidad de Guadalajara, México. Ha obtenido el grado de Doctora por la Universidad de Granada, España, con mención de Doctorado Internacional dentro del Programa Oficial de Doctorado en Ciencias de la Educación. Sus líneas de investigación corresponden al campo de la Internacionalización de la Educación Superior. Tiene experiencia en organización de diplomados conjuntos con universidades extranjeras y como formadora de formadores en temas de Alfabetización Informacional (ALFIN). Recientemente se ha integrado como colaboradora en actividades de evaluación y dictamen de artículos científicos. mariceladelaisla@hotmail.com

\section{Antonio Humberto Closas}

Se desempeña como Profesor Titular de Estadística II y Director de Proyectos de Investigación en la Facultad de Ciencias Económicas de la Universidad Nacional del Nordeste, esta última función también la ejerce en la Facultad Regional Resistencia de la Universidad Tecnológica Nacional, ambas Instituciones de Argentina. Actualmente ejerce el cargo de vicepresidente primero de la Sociedad Argentina de Estadística. Ha obtenido el grado de Doctor en la Universidad Pública de Navarra, España, en el área de Estadística e Investigación Operativa. Sus líneas de investigación están asociadas con métodos estadísticos multivariantes aplicados a estudios sociales y económicos. Es coautor de libros y de diversas publicaciones en revistas de impacto científico nacionales y extrajeras. Es investigador categorizado del Programa de Incentivos, de la Secretaría de Políticas Universitarias, del Ministerio de Educación de la Nación, así como de la Secretaría de Ciencia, Tecnología y Posgrado de la Universidad Tecnológica Nacional. Ha realizado estancias de investigación en Universidades de Europa y de Latinoamérica. Participa como conferencista y expositor en reuniones académicas que se realizan tanto en Argentina, como en el exterior. Asimismo, es integrante de distintos comités de arbitraje de revistas científicas y de comisiones evaluadoras de tesis de maestrías y doctorados.

hclosas@hotmail.com

\section{Antonio Luzón Trujillo}

Es Profesor Titular de Educación Comparada e Internacional de la Universidad de Granada (España). Es presidente de la Sociedad Española de Educación Comparada (SEEC) y miembro de la Comparative Education Society in Europe, (CESE). Coordinador del Grupo de Investigación "Políticas Educativas y Reformas" de la Junta de Andalucía (España), desde donde colabora y participa en diversos proyectos de investigación, nacionales, “Análisis y evaluación de los programas de 
Formación Profesional Básica en la transición de la juventud a la vida laboral y adulta" (Proyecto Nacional I+D) y europeos "Policies Supporting Young Adults in their Life Course. A Comparative Perspective of Lifelong Learning and Inclusion in Education and Work in Europe" (Programa Horizon 2020), vinculados a la educación y políticas comparadas. Es director del Máster Universitario en "Investigación, desarrollo social e intervención socioeducativa”, vinculado al Master Erasmus Mundus "MITRA" y director de la cátedra "Educación y Sociedad” en la misma Universidad. Ha sido profesor visitante en universidades europeas (Francia, Italia) y latinoamericanas (México, Chile, Brasil). Es autor de numerosas publicaciones relacionadas con la política educativa.

aluzon@ugr.es 